\title{
Characterization of viroplasm formation during the early stages of rotavirus infection
}

\author{
José J Carreño-Torres, Michelle Gutiérrez, Carlos F Arias, Susana López, Pavel Isa
}

\begin{abstract}
Background: During rotavirus replication cycle, electron-dense cytoplasmic inclusions named viroplasms are formed, and two non-structural proteins, NSP2 and NSP5, have been shown to localize in these membrane-free structures. In these inclusions, replication of dsRNA and packaging of pre-virion particles occur. Despite the importance of viroplasms in the replication cycle of rotavirus, the information regarding their formation, and the possible sites of their nucleation during the early stages of infection is scarce. Here, we analyzed the formation of viroplasms after infection of MA104 cells with the rotavirus strain RRV, using different multiplicities of infection $(\mathrm{MOl})$, and different times post-infection. The possibility that viroplasms formation is nucleated by the entering viral particles was investigated using fluorescently labeled purified rotavirus particles.

Results: The immunofluorescent detection of viroplasms, using antibodies specific to NSP2 showed that both the number and size of viroplasms increased during infection, and depend on the MOI used. Small-size viroplasms predominated independently of the $\mathrm{MOI}$ or time post-infection, although at MOl's of 2.5 and 10 the proportion of larger viroplasms increased. Purified RRV particles were successfully labeled with the Cy5 mono reactive dye, without decrease in virus infectivity, and the labeled viruses were clearly observed by confocal microscope. PAGE gel analysis showed that most viral proteins were labeled; including the intermediate capsid protein VP6. Only 2 out of 117 Cy5-labeled virus particles colocalized with newly formed viroplasms at 4 hours post-infection.
\end{abstract}

Conclusions: The results presented in this work suggest that during rotavirus infection the number and size of viroplasm increases in an MOl-dependent manner. The Cy5 in vitro labeled virus particles were not found to colocalize with newly formed viroplasms, suggesting that they are not involved in viroplasm nucleation.

\section{Background}

Rotaviruses are the major cause of severe diarrhea in children and young animals worldwide. As a members of the family Reoviridae, they have a genome of $11 \mathrm{seg}$ ments of double-stranded RNA (dsRNA) enclosed in three protein layers, forming infectious triple-layered particles (TLP) [1]. During, or just after entering the cell's cytoplasm, the outer capsid, composed of VP4 and VP7, is released, yielding transcriptionally active doublelayered particles (DLP). The produced viral transcripts direct the synthesis of viral proteins and serve as templates for the synthesis of negative-RNA strands to form the genomic dsRNA. During the replication cycle of rotavirus electron-dense cytoplasmic inclusions, named viroplasms, are formed [2]. Such cytoplasmic inclusions

\footnotetext{
* Correspondence: pavel@ibt.unam.mx

Departamento de Genética del Desarrollo y Fisiología Molecular, Instituto de Biotecnología, Universidad Nacional Autónoma de México
}

are observed during infection with a number of animal viruses [3], including reoviruses, as other members of the Reoviridae family [4].

In rotaviruses two non-structural proteins, NSP2 and NSP5, have been shown to be sufficient to form membrane-free cytoplasmic inclusions, which are known as viroplasms-like structures [5]. In vivo immunofluorescence visualization of viroplasms shows they are heterogeneous in size [6,7]. It is in these structures where the synthesis of dsRNA and its packaging into pre-virion core particles take place [8]. Besides NSP2 and NSP5, other viral proteins accumulate in viroplasms - namely VP1, VP2, VP3, VP6, and NSP6 [7,9-11]. The key role of NSP2 and NSP5 proteins in the formation of viroplasms has been demonstrated by knocking-down their expression by RNA interference, which results in the inhibition of viroplasm formation, genome replication, virion assembly, and a general decrease of viral protein 
synthesis $[7,8,12]$. Viroplasm formation has been studied using electron or fluorescence microscopy [6,13-15], however, despite their importance in the replication cycle of rotavirus, little is know about their dynamics of formation. The observation that bromouridine-labeled RNA localizes to viroplasms suggested that the viral transcripts are synthesized within viroplasms, which led to the hypothesis that the entering viral particles could serve as points of nucleation for the formation of viroplasms [8]. In this work, the dynamics of viroplasm formation in MA104 cells infected with rotavirus strain RRV was studied as a function of time and multiplicity of infection (MOI). Using fluorescently labeled purified rotavirus particles; we showed that the incoming TLPs do not seem to be involved in the formation of viroplasms.

\section{Materials and methods \\ Cells, viruses, antibodies, and fluorophores}

MA104 cells were cultured in Advanced Dulbecco's Modified Eagle's Medium (DMEM) supplemented with $3 \%$ fetal calf serum (FBS). The rhesus rotavirus strain RRV, obtained from H.B. Greenberg (Stanford University, Stanford CA), was propagated in MA104 cells. The rabbit polyclonal serum to NSP2 protein has been described previously [16]. Horseradish peroxidaseconjugated goat anti-rabbit polyclonal antibody was from Perkin Elmer Life Sciences (Boston, MA), Alexa 488 and 568 -conjugated goat anti-rabbit polyclonal antibodies, FluoSpheres carboxylate-modified microspheres, $0.1 \mu \mathrm{m}$, yellow-green fluorescent (505/515), were from Molecular Probes (Eugene, OR), and $\mathrm{Cy}^{\mathrm{mm}} 5$ Mono-Reactive Dye pack was from Amersham, GE Healthcare, UK.

Identification, quantitation and size analysis of viroplasms MA104 cells grown in $10 \mathrm{~mm}$ coverslips were infected with rotavirus strain RRV at different MOI's for 1 hour at $4^{\circ} \mathrm{C}$. After washing unbound virus, the cells were incubated at $37^{\circ} \mathrm{C}$ for different times post-infection. The cells were fixed with $2 \%$ paraformaldehyde, and permeabilized with $0.5 \%$ Triton X-100 in PBS containing 1\% bovine serum albumin, as described previously [17]. Cells were then incubated with rabbit polyclonal sera to NSP2 protein, followed by staining with goat anti-rabbit IgG coupled to Alexa-488 or 568 . The images were acquired using a Zeiss Axioskop 2 Mot Plus microscope and analyzed by Image Pro Plus 5.0.2.9 and Adobe Photoshop 7.0. All images were acquired with a $60 \times$ objective, with a real time CCD Camera in 256 grey scales, and the size of the images was $1392 \times 1040$ pixels, with 8 bits. The estimation of viroplasm size was done using the Analyze particle function of Image J 1.32j program (Wayne Rasband, NIH, USA).

\section{Immunodetection of rotavirus NSP2 protein}

MA104 cells grown in 24-well plates were infected with rotavirus strain RRV at different MOI's for 1 hour at $4^{\circ} \mathrm{C}$. After washing unbound virus, the cells were incubated at $37^{\circ} \mathrm{C}$ for different times post-infection. At the indicated time points, the cells were washed twice with PBS and lysed with Laemmli sample buffer. Proteins were separated by $10 \%$ SDS-PAGE and transferred to nitrocellulose membranes (Millipore, Bedford, MA). Membranes were blocked with $5 \%$ non-fat dried milk in PBS, and incubated at $4^{\circ} \mathrm{C}$ with primary anti NSP2 polyclonal antibody in PBS with $0.1 \%$ milk, followed by incubation with secondary, horseradish peroxidase-conjugated antibodies. The peroxidase activity was revealed using the Western Lightning ${ }^{\text {Tw }}$ Chemiluminiscence Reagent Plus (Pelkin Elmer Life Sciences). The images obtained were scanned and the band densities analyzed using Image pro software.

\section{Conjugation of virus with fluorophore and colocalization of labeled viruses with viroplasms}

To label virus with fluorophores, RRV virions were purified by cesium chloride gradient centrifugation as described previously [18]. The purified TLP's of simian strain RRV were washed twice with $10 \mathrm{mM}$ Hepes $\mathrm{pH}$ 8.2, $5 \mathrm{mM} \mathrm{CaCl}_{2}, 140 \mathrm{mM} \mathrm{NaCl}$, and labeled with Cy5 mono reactive dye $(0.1,0.5,1,2.5$, and $5 \mathrm{nmol}$ of fluorophore for $1 \mu \mathrm{g}$ of purified virus) at room temperature for 1 hour with gentle agitation. The reaction was stopped by addition of Tris- $\mathrm{HCl} \mathrm{pH} 8.8$ to a final concentration of $50 \mathrm{mM}$. Labeled viruses were separated from unbound fluorophore by gel filtration on a G25 sepharose column. As control, the purified TLP's of strain RRV were processed in identical way without addition of fluorophore. Viral titres were determined by a standard immunoperoxydase assay as described previously [19]. DLP's were prepared by EDTA treatment of labeled TLP's. To determine which viral proteins were conjugated with fluorophore, labeled and nonlabeled TLP's and DLP's were resolved in PAGE gel, analyzed on Typhoon-Trio (Amersham Biosciences) and stained by silver nitrate. Labeled particles were compared with FluoSpheres [carboxylate-modified microspheres, $0.1 \mu \mathrm{m}$, yellow-green fluorescent (505/515)] using confocal microscope LSM-510 Zeiss, mounted on inverted microscope Zeiss Axiovert 200 M, with AIM software, using objective Plan-neofluor $100 \times / 1.30$ Oil Ph3 (Carl Zeiss). To detect green staining, excitation laser Argon $2488 \mathrm{~nm}$ was used with emission filter BP 500-530 nm, and for far red staining laser Helio-Neon $633 \mathrm{~nm}$ was used with emission filter BP650-670 nm. To colocalize labeled TLP's with viroplasms, MA104 cells grown on coverslips were infected with Cy5 -labeled RRV TLP's (MOI of 2) for 1 hour at $37^{\circ} \mathrm{C}$. 
After washing unbound virus, the infection was left to proceed for 4 hours, and then the cells were fixed and the viroplasms were detected as described above using a rabbit polyclonal antibody specific for rotavirus NSP2 protein, and a goat anti rabbit IgG coupled to Alexa 488. Images were acquired using a confocal microscope as described above, as stacks of 10 images $800 \mathrm{~nm}$ thick, with resolution of $1024 \times 1024$ pixels, and processed by nearest neighbor deconvolution using AIM software. Acquired images were processed by Image J 1.32j and Adobe Photoshop 7.0. Analyzing corresponding individual images ensured localization of all $\mathrm{Cy}-5$ labeled viral particles inside cytoplasm.

\section{Results}

The number of viroplasms and the level of the NSP2 protein increase during rotavirus infection, in direct correlation with the multiplicity of infection

It has been described that viroplasms can be visualized in rotavirus infected cells by immunofluorescence as early as 2 hours postinfection (hpi) [14]. Therefore, to learn about the kinetics of viroplasm formation at early stages of rotavirus infection, MA104 cells grown in coverslips were infected with rotavirus strain RRV at different MOI's, and at different times post-infection $(2,4,6$, or 8 hours) the cells were fixed and the viroplasms were detected by immunofluorescence using a mono-specific serum to NSP2. Viroplasms were detected as early as 2 hpi as discrete dots that were not observed in control, mock infected cells, and their number and size increased as the infection proceeded (Figure 1). To quantitate the increase in the number of viroplasms during infection, the number of viroplasms in 400 infected cells from each condition was scored. It was observed that independently of the MOI used, the number of viroplasms per cell increased as the infection proceeded $(\mathrm{P}<0.05$, Student's t-test), almost duplicating every two hours up to 6 hours (Figure 2).

To determine the size of viroplasms, their area was determined in pixels ${ }^{2}$ in 360 infected cells per condition. While at higher MOI's (2.5 and 10) there was a constant increase in the average size of the viroplasms, at low MOI's (0.1 and 0.5) a fluctuation in the viroplasm size was observed (Figure 3A). To analyze the size of the viroplasms in more detail, viroplasms were divided into three arbitrary groups: small ( $4-33$ pixels $\left.^{2}\right)$, medium $\left(34-69\right.$ pixels $\left.^{2}\right)$, and large $\left(70-200\right.$ pixels $\left.^{2}\right)$ (Figure 3B). Throughout the course of infection, and independently of the MOI used, or the time post infection analyzed, the population of small viroplasms predominated in number and also in proportion of all viroplasms (Figure 3C). Differences were observed when the size of viroplasms was compared in cells infected with low (0.1 and 0.5$)$ or high (2.5 or 10) MOI's. While at high MOI's there was a gradual decrease in the proportion of small viroplasms during the course of infection, from 90 and $92 \%$ ( $2 \mathrm{hpi}$ ) to 56 and $45 \%$ (8 hpi) respectively, with a concomitant increase in the medium and large size viroplasms, the proportion of small viroplasms at low MOI's was more stable (Figure $3 \mathrm{C}$ ). This suggests that while at high MOI's small viroplasms might convert to larger size viroplasms, probably due to large amount of protein synthesized in cell, at low MOI's the formation of new small viroplasms prevails, and they could become larger at later times post infection, however, this possibility was not investigated in this work.

To determine if the number and size of viroplasms correlate with the level of NSP2 synthesized during infection, cells infected at different MOI's were harvested at different times post-infection, and the level of NSP2 was determined by Western blot (Figure 4). While at high MOI's (2.5 and 10) the NSP2 protein was detected at 4 hours post-infection, and increased as infection proceeded, at low MOI's (0.1 and 0.5) the NSP2 protein was detected until 8 hours post-infection (Figure 4A). Densitometric analyses showed that the dynamics of accumulation of NSP2 during infection with high MOI's (Figure, 4B) was similar to that observed for the increase in the number of viroplasms (Figure 2).

\section{Viroplasms do not colocalize with fluorescently labeled particles}

It has been previously described that virus-like particles produced in insect cells by the co-expression of rotavirus capsid proteins VP6, VP4, VP7, and a VP2 protein fused to GFP or to DsRed protein, can be visualized in living cells [20]. Other viruses have also been observed in cells after being directly labeled with fluorophores; among these are influenza $A$ virus, poliovirus, dengue virus, and SV40 [21-24]. To determine if the formation of viroplasms is nucleated by the entering viral particles, purified infectious TLP's of RRV were conjugated with Cy5. Analysis of viral proteins by PAGE showed that all proteins were conjugated. Importantly the intermediate capsid protein VP6 was efficiently labeled, ensuring that viral particles will be visible even after loss of the outer capsid proteins VP7 and VP4 (or its trypsin cleavage products VP5 and VP8) (Figure 5A and 5B). Viral titre after conjugation was similar to mock conjugated virus, observing a small decrease of infectivity when using 5 $\mathrm{nmol}$ of $\mathrm{Cy} 5$ for conjugation, suggesting that viral infectivity was not compromised (Figure 5C), therefore, for the following experiments $1 \mathrm{nmol}$ of $\mathrm{Cy} 5 / \mu \mathrm{g}$ of virus was chosen. Most importantly, both TLP's and DLP's (prepared from TLP's by EDTA treatment), labeled with $1 \mathrm{nmol}$ of $\mathrm{Cy} 5$, were comparable to $100 \mathrm{~nm}$ Fluorospheres when observed in confocal microscopy 


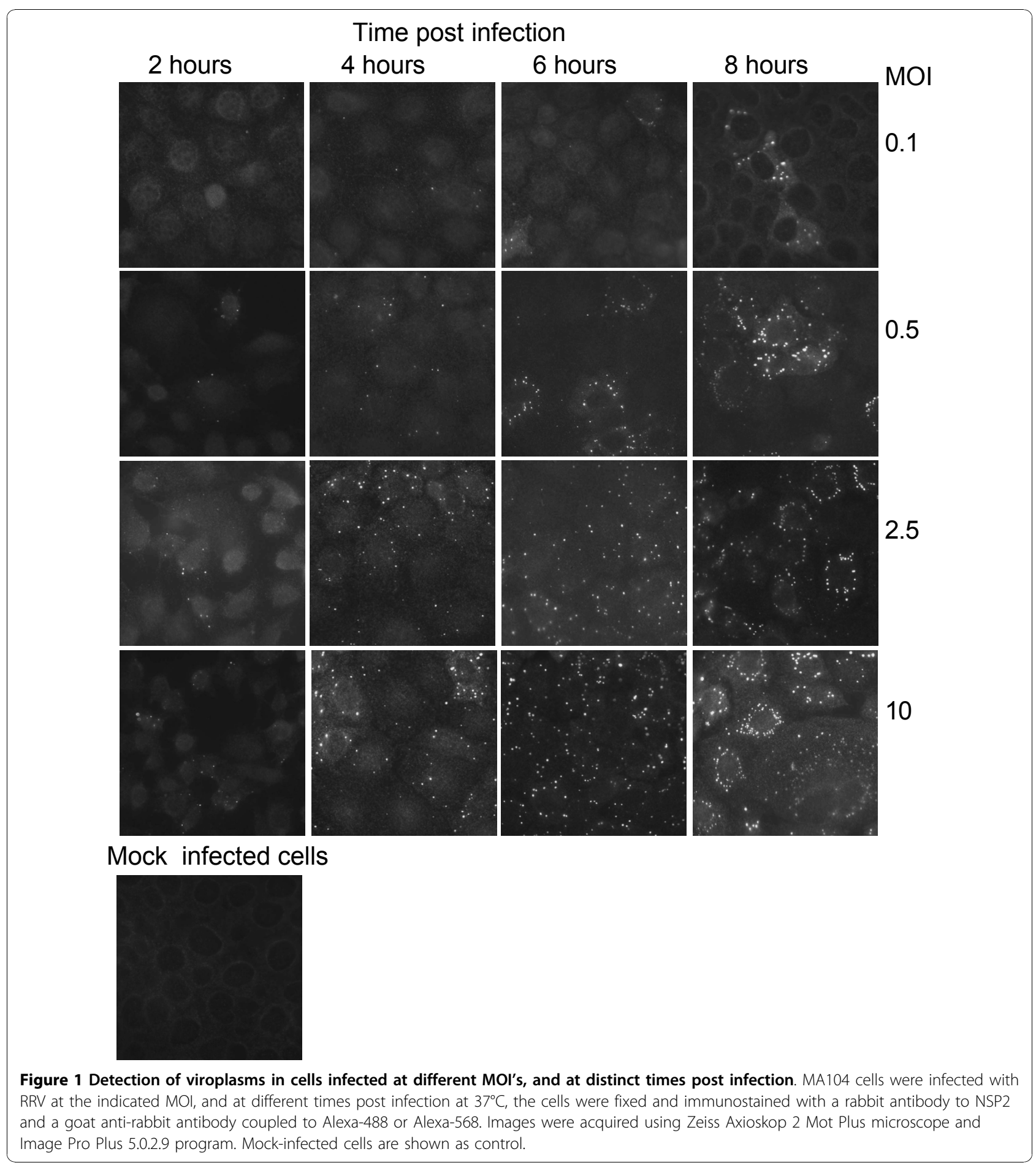

(Figure 5D). Since it was possible to visualize the fluorescently labeled viral particles, we used them to observe their intracellular distribution with respect to the newly formed viroplasms. To do this, MA104 cells grown in coverslips were infected with Cy5-conjugated RRV TLP's at an MOI of 2, and 4 hpi the cells were fixed, the viroplasms were immunostained using a polyclonal sera to NSP2, and images were acquired using confocal microscopy, as described under material and methods. Fluorescently labeled viral particles were observed distributed in the cytoplasm as discrete spots (Figure 6). The number of labeled viral particles, viroplasms, and their co-localization was counted independently by two persons in 31 cells. In these, 117 labeled virus particles 


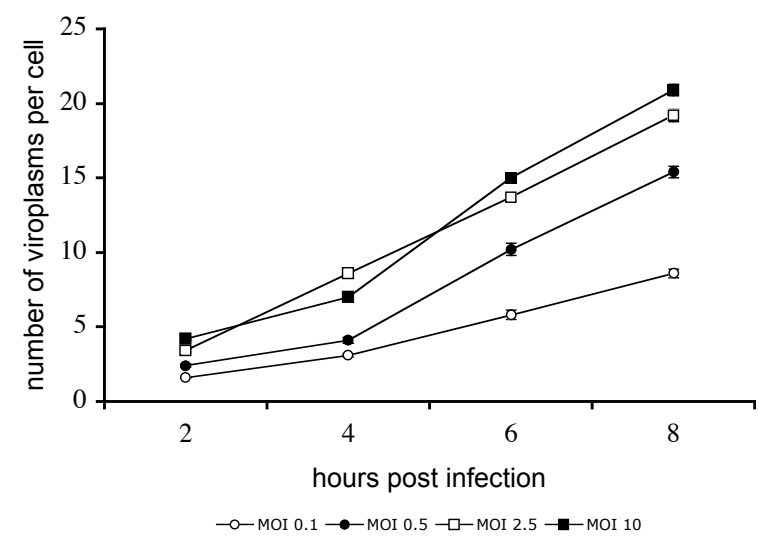

Figure 2 The number of viroplasms per cell increases with time of infection. MA104 cells were infected at different MOl's as described in Figure 1, and viroplasms were detected by immunofluorescent staining of NSP2. Viroplasms were counted in 400 infected cells in each condition. Each value is expressed as mean \pm standard error. The increase in the number of viroplasms during the infection at each $\mathrm{MOl}$, and the differences in the number of viroplasms between different $\mathrm{MOI}$ at each time point were statistically significant $(P<0.05$, student T-test).

and 467 viroplasms were observed, however, only 2 of the viral particles observed colocalized with viroplasms, while the rest appeared independent of each other in the cell cytoplasm.

\section{Discussion}

The formation of viroplasms has been previously studied using electron and fluorescence microscopy, however, those studies have focused only on late (4 to $24 \mathrm{hpi}$ ) stages of infection $[6,13,15]$. Only Eichwald et al [14] have studied earlier stages of viroplasm formation, and in their work, following the expression of an NSP2 protein fused to EGFP in rotavirus SA-11 infected cells, they observed that the total number of viroplasms decreased with time, with a concomitant increase in their size, starting at $6 \mathrm{hpi}$. This observation was interpreted as fusion events between smaller viroplasms. Similar results were reported by Cabral-Romero and Padilla-Noriega [15] using the strain SA-11 in BSC1 cells, although at even later (10 hpi) stages of infection. Comparing the formation of viroplasms between SA-11 and OSU rotavirus strains, Campagna et al. [6] observed that the viroplasms formed in OSU infected cells did not increase in size as readily as those formed during infection with SA-11. In this work, after infection with rotavirus strain RRV, using different MOI's, an increase in the number of viroplasms and in the amount of the NSP2 protein was observed. The size of viroplasms was observed to increase when higher MOI's were used.

There are several possibilities to explain the discrepancies reported. First, the decrease in the number of viroplasms was observed only during infection with strain SA-11 [14,15], but not with strains OSU [12], and RRV (this work). It is known that some viral functions (receptor specificity, plaque formation, extraintestinal spread, IRF3 degradation, etc) may vary among different rotavirus strains [25-28] what opens the possibility that there could also be strain-specific differences for viroplasm formation. In fact, an impaired phosphorylation of NSP5 affected differently the morphogenesis of viroplasms in cells infected with either SA-11 or OSU rotavirus strains [6]. The differences observed between our studies and those of other groups could also arise from the different methodologies used to detect viroplasms. While in our case the newly synthesized rotavirus proteins were immunodetected and analyzed in 400 cells, in the study by Eichwald et al. [14] the identification of viroplasms was based on the detection of NSP2-EGFP or NSP5-EGFP fusion proteins in 20 cells. It is possible that the large amount of recombinant fusion proteins that accumulated in the cytoplasm of transfected cells before rotavirus infection could change the kinetics of viroplasm formation, since upon rotavirus infection a rapid redistribution of the EGFP - proteins was observed. It was not possible to compare the exact number of viroplasms obtained in that study, since the MOI that was used to infect the transfected MA104 cells was not mentioned.

In this work, studying the kinetics of viroplasm formation during the infection of strain RRV, we observed an increase in both the number and size of viroplasms with time and this increment was dependent on the MOI used. At high MOI's (2.5 and 10) the increase correlated with the amount of NSP2 protein detected at a given time point, while at lower MOI's (0.1 and 0.5), the smaller increase in NSP2 protein correlated with a less variable viroplasm size. It is possible that when a critical concentration of NSP2 and NSP5 is reached, and as other viral proteins accumulate, viroplasms start to form, first as small entities, and then becoming larger at later stages of the replication cycle. Although, it is not possible to determine if the increase in size is caused by fusion of smaller viroplasms or by addition of newly produced rotavirus proteins to small viroplasms, our observations are more consistent with the idea that new small viroplasms are generated constantly during the replication cycle, since even at later stages of infection a large proportion of small viroplasms was observed. It remains to be determined if the small viroplasms, presumably generated by the aggregation of NSP2 and NSP5 require an additional priming signal, or if it is only the concentration of free NSP2 and NSP5 what dictates the formation of a new viroplasms.

The mechanism of viroplasms formation and its protein content is unknown. The fact that viroplasms are sites for 
A

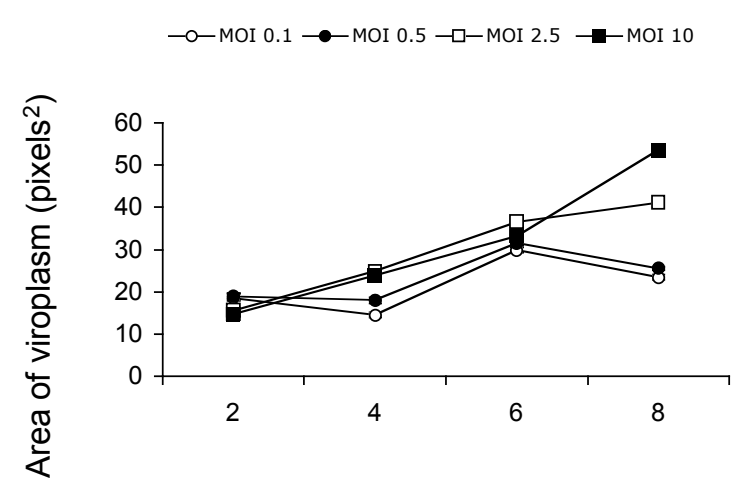

hours post infection

C

MOI 0.1
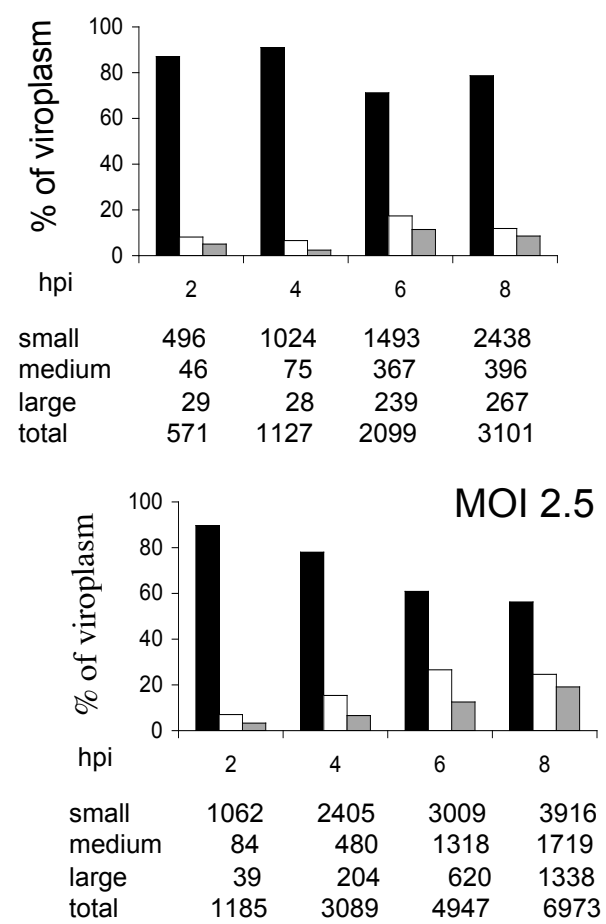

B

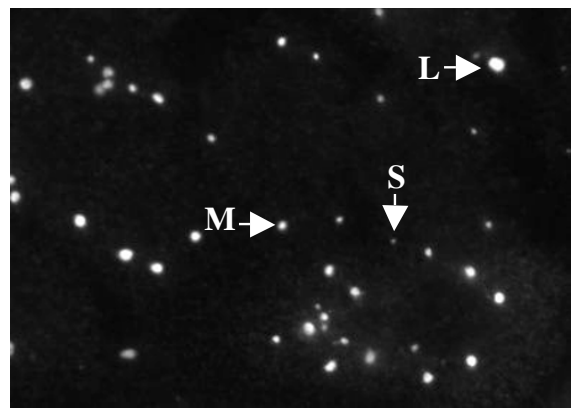

MOI 0.5

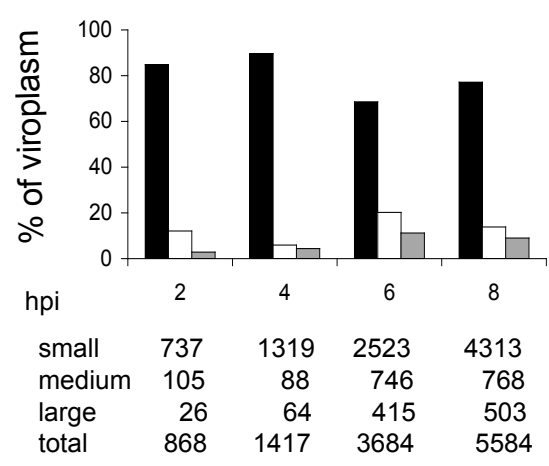

MOI 10

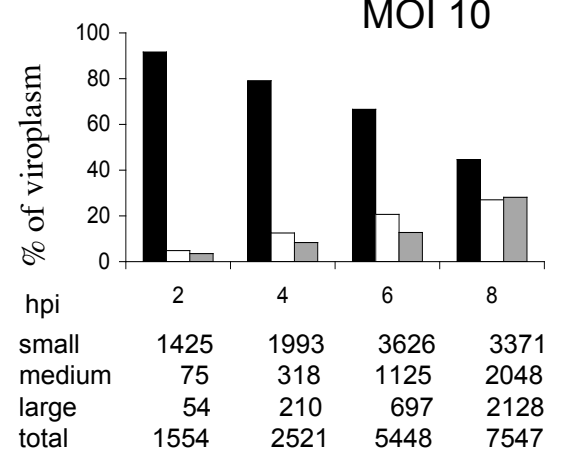

Figure 3 The proportion of larger viroplasms increases during rotavirus infection. (A) MA104 cells were infected at different MOl's as described in Figure 1, and the area of each viroplasm was estimated by pixel determination using Image J. The same images used in Figure 2 were analyzed for this figure. Each value represents the mean \pm standard error of viroplasms detected in 360 cells, in pixels ${ }^{2}$. (B) Based on a microscopic comparison, viroplasms were divided into three arbitrary groups, small (S) (4-33 pixels $\left.{ }^{2}\right)$, medium (M) (34-69 pixels $\mathrm{s}^{2}$, and large (L) (70-200 pixels ${ }^{2}$ ). Arrows point to viroplasms representative of each size $\mathrm{S}, \mathrm{M}$, and L. (C) Relative amounts of small, medium and large viroplasms during the course of infection at different MOl's. Bars represent the proportion of viroplasms for each multiplicity of infection, (black bars - small; white bars medium; grey bars large viroplasms) with 100\% being the total number of viroplasms counted in 360 cells. The numbers under each graph represent the number of the different classes of viroplasms found in each condition analyzed.

rotavirus transcription at late stages of infection $(8.5 \mathrm{hpi})$ led to the so far unproven hypothesis that incoming DLP's serve as focal points of viroplasm assembly [8]. In this work we tested this hypothesis by visualization of incoming viral particles and by analyzing their colocalization with newly formed viroplasms. Only 2 out of 117 CY5- conjugated viral particles observed in 31 cells colocalized with viroplasms, suggesting that the entering virus particles do not serve as focal points for accumulation of the newly synthesized proteins into viroplasms.

In addition, if the entering virus particles served as focal point for viroplasm formation, the number of 
A

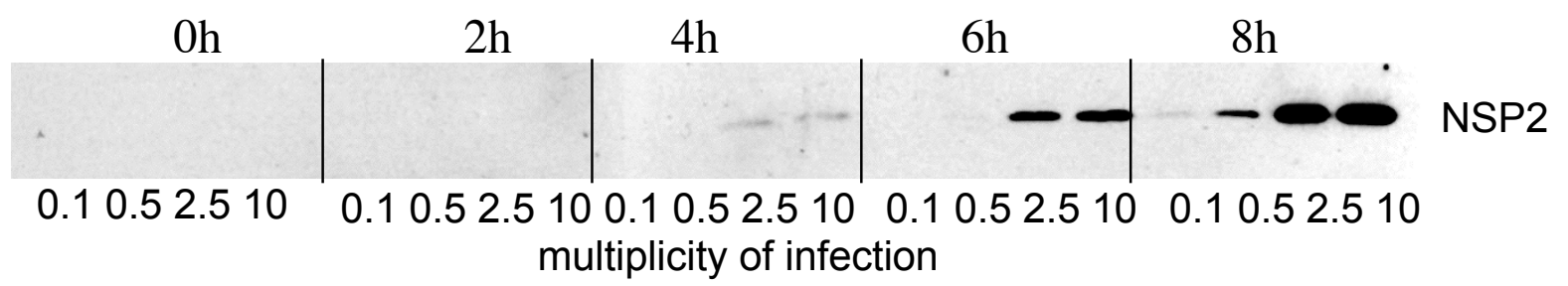

B

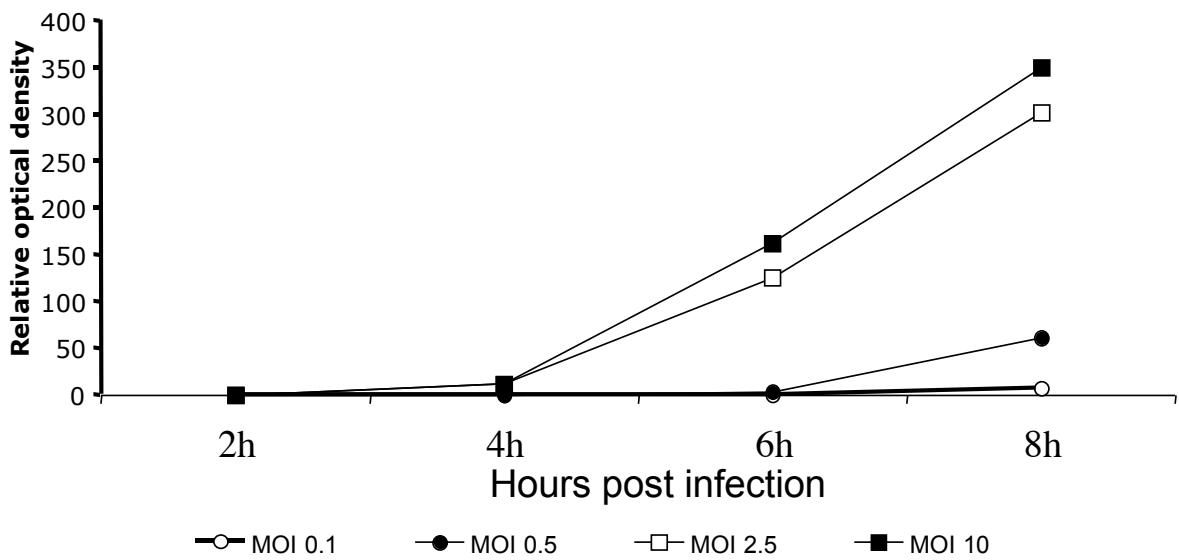

Figure 4 The amount of NSP2 protein increases with time of infection. MA104 cells were infected with RRV at the indicated MOI, and at different times post-infection at $37^{\circ} \mathrm{C}$, the cells were harvested in Laemmli buffer. Equal amount of cell lysates were separated by SDS-PAGE and blotted onto nitrocellulose. (A) The expression of rotavirus NSP2 protein was determined by immunostaining with a rabbit antibody to NSP2 and a goat anti-rabbit antibody coupled to peroxydase. A representative experiment from three carried out is shown. (B) Optical density of the

protein bands shown in A, as determined by scanning and analysis using Image pro.

viroplasms at early times of infection should correspond to the estimated number of infectious viral particles that entered the cell. However, a correlation between the number of viroplasms detected at early times post-infection and the expected number of infectious particles entering the cells, according to the Poisson distribution (Table 1), was not observed (Figure 2). At low MOI's, when $95 \%$ and $77 \%$ of infected cells are expected to be infected with only 1 viral particle (with MOI's of 0.1 and 0.5 respectively), there were more viroplasms per cell [1.6 and 3.1 for a MOI of 0.1 and 2.4 and 4.1 for an MOI of 0.5 ( 2 and 4 hpi respectively)], while at an MOI of 10 , when $87 \%$ of the cells are expected to be infected with 7 or more infectious viral particles, only 4.2 and 7 viroplasms were observed at 2 and 4 hours post-infection (Figure 2). These results suggest that at the onset of infection the entering viral particles do not serve as nucleation centers for the formation of viroplasms as suggested [8]. The fact that the plasmid expression of NSP2 and NSP5 proteins alone, in absence of infectious virus, are able to form viroplasm-like structures also supports this conclusion.

Recently it was suggested that rotavirus viroplasms could interact with microtubules [15]. NSP2 was also shown to interact with tubulin, inducing the collapse of the microtubule network, and viroplasms were shown to colocalize with tubulin granules [29]. Similar interaction of reovirus viral inclusion bodies with microtubules [30] suggests the possibility that tubulin could have a more general role in the replication cycle of viruses of the Reoviridae family.

Although viroplasms play a crucial role in rotavirus replication and assembly, the factors that govern their formation and function, are still not clearly understood. The development of live cell imaging tools should provide more detailed information about these processes.

\section{Conclusions}

Rotavirus replication takes place in electrodense structures known as viroplasms, however, little is known about their dynamics of formation, and the factors that drives viroplasm nucleation. The results presented in this work show that during rotavirus infection the number and size of viroplasms increases steadily with time, and depends on the MOI used. Using in vitro Cy5 labeled infectious viral particles we observed that the entering viruses do not seem to be involved in viroplasm nucleation. It is possible that some cellular protein, like 


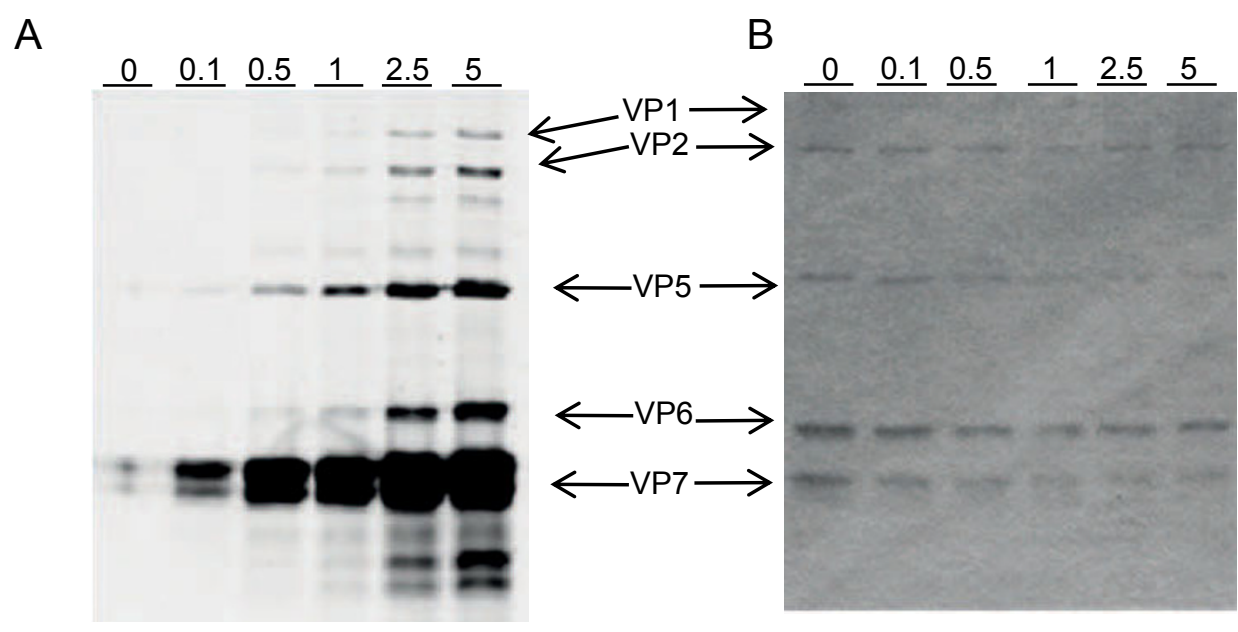

C

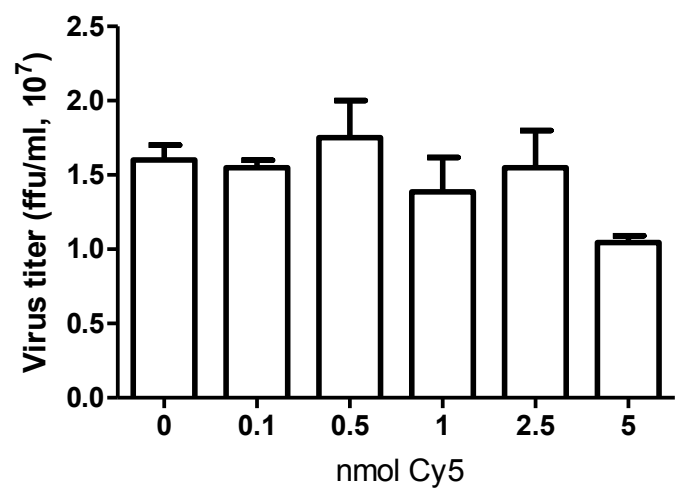

D

\section{spheres}

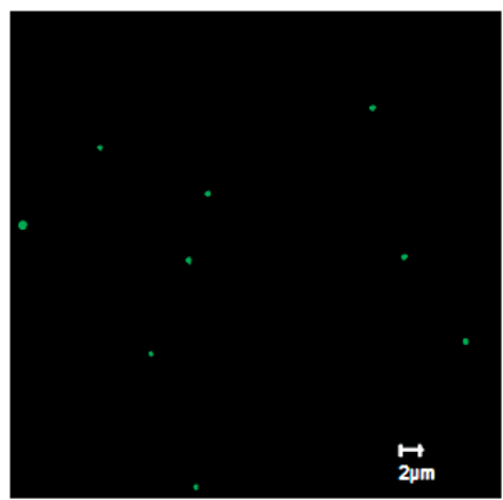

Cy5 TLPs

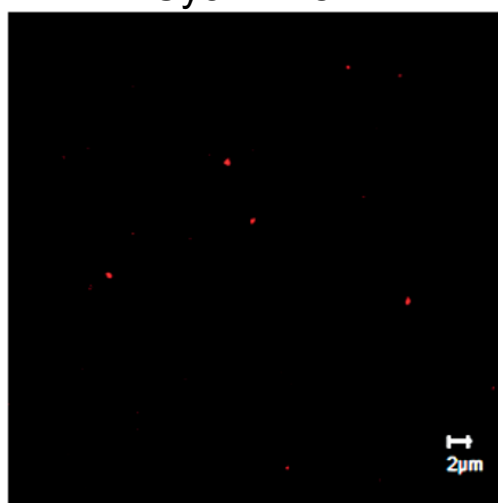

\section{Cy5 DLPs}

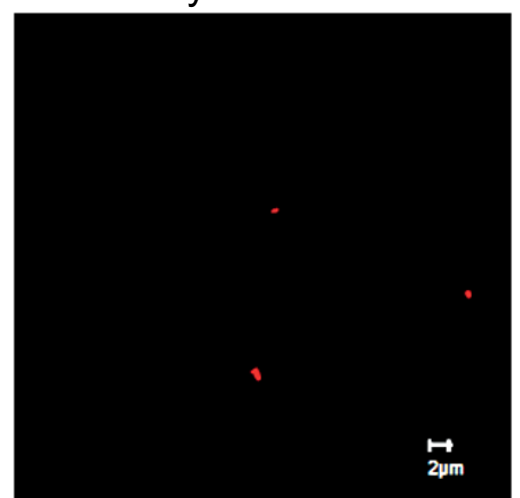

Figure 5 Conjugation of viral particles with Cy5 monoreactive dye. Purified TLP's of strain RRV were conjugated with different amounts of Cy5 monoreactive dye as described under Materials and methods. The reaction was stopped by Tris- $\mathrm{HCl}$ and labeled viruses were separated by gel filtration on G25 sepharose column. (A) Labeled and non-labeled viral particles were separated on 10\% PAGE, and gel was visualized on Typhoon Trio to determine Cy5 - viral protein conjugation. (B) Same gel as shown in A was stained using silver nitrate. Viral proteins are identified by arrows. (C) MA104 cells grown in 96 well plates were infected with labeled and non labeled viral preparations, and 14 hours post infections cells were fixed and infected cells were detected using peroxydase immuno staining with anti-rotavirus polyclonal antibodies. Results are expressed as number of viral infectious focus forming units per ml. (D) Comparison of fluorophore labelled TLP's and DLP's (prepared by EDTA treatment), shown in red, with $100 \mathrm{~nm}$ Fluorosferes, shown in green, observed in confocal microscope. 


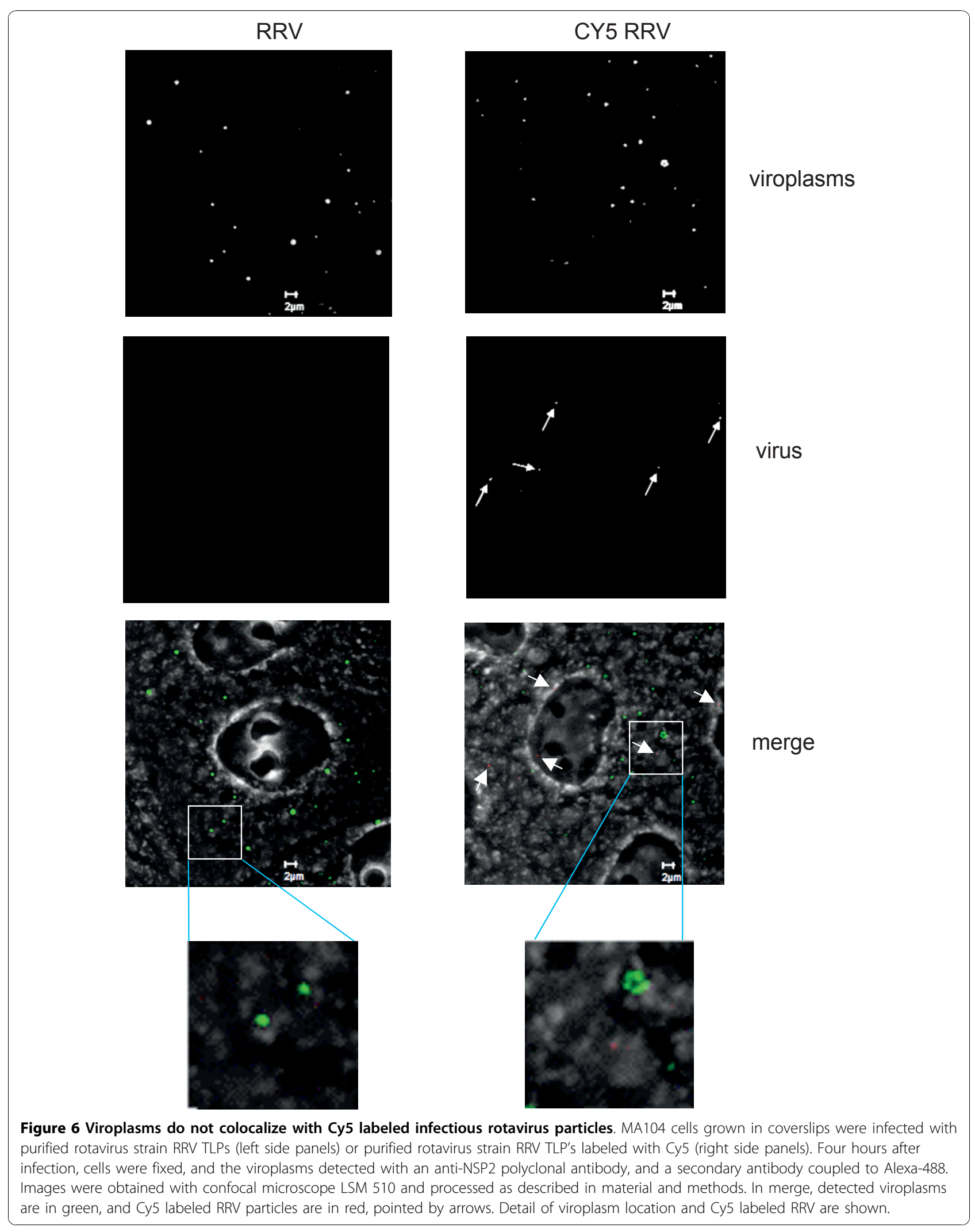


Table 1 Theoretical percentage of cells infected with a given number of viral particles at different multiplicities of infection, as determined by the Poisson distribution, with $100 \%$ being all infected cells.

\begin{tabular}{ccccc}
\hline & \multicolumn{5}{c}{ Multiplicity of infection } \\
No. of infectious viral particles/cell & $\mathbf{0 . 1}$ & $\mathbf{0 . 5}$ & $\mathbf{2 . 5}$ & $\mathbf{1 0}$ \\
\hline 1 & $95.1^{*}$ & 77.1 & 22.4 & 0.05 \\
2 & 4.7 & 19.3 & 27.9 & 0.2 \\
3 & 0.2 & 3.2 & 23.3 & 0.8 \\
4 & 0.003 & 0.4 & 14.6 & 1.9 \\
5 & $0+$ & 0.04 & 7.3 & 3.8 \\
6 & $0+$ & 0.003 & 3.0 & 6.3 \\
7 & $0+$ & 0.0002 & 1.1 & 9.0 \\
8 & $0+$ & $0 \dagger$ & 0.3 & 11.3 \\
9 & $0+$ & $0+$ & 0.09 & 12.5 \\
10 & $0+$ & $0+$ & 0.02 & 12.5 \\
11 & $0+$ & $0+$ & 0.005 & 11.4 \\
12 & $0+$ & $0+$ & 0.001 & 9.5 \\
13 & $0+$ & $0 \dagger$ & 0.0002 & 7.3 \\
14 & $0+$ & $0 \dagger$ & $0 \dagger$ & 5.2 \\
$\geq 15$ & $0+$ & $0 \dagger$ & $0 \dagger$ & 8.3 \\
$\%$ of total cells infected & 9.5 & 39.3 & 91.8 & 99.8 \\
\hline
\end{tabular}

$* \%$ of infected cells
$\dagger<0.0001 \%$

tubulin, are required for this process, however much work is needed to characterize in detail this essential step of rotavirus replication.

\section{List of abbreviations}

DLP: double layered particles; DMEM: dulbecco's modified eagle medium; DSRED: Discosoma sp. Red fluorescence protein; DSRNA: double stranded RNA; EGFP: enhanced green fluorescence protein; GFP: green fluorescence protein; HPI: hours post infection; IRF3: interferon regulatory factor 3; MOI: multiplicity of infection; NSP2: nonstructural protein 2; NSP5: nonstructural protein 5; NSP6: nonstructural viral protein 6; PAGE: polyacrylamide gel electrophoresis; TLP: triple layered particles; VP1: structural viral protein 1; VP2: structural viral protein 2; VP3: structural viral protein 3; VP4: structural viral protein 4; VP5: structural viral protein 5; VP6: structural viral protein 6; VP7: structural viral protein 7; VP8: structural viral protein 8.

\section{Acknowledgements}

We acknowledge the excellent technical assistance of M.C. Andres Saralegui Amaro with confocal microscopy and Pedro Romero for virus purification. This work was partially supported by grants 55005515 from the Howard Hughes Medical Institute, grant 60025 from CONACYT, Mexico, and IN210807 from DGAPA-UNAM. JJCT and MG were recipients of a scholarship from CONACYT.

\section{Authors' contributions}

JJCT carried out study of kinetics of viroplasms formation, started analysis of fluorophore conjugated viral particles, MG carried out Cy5-TLP's: viroplasm colocalization studies, CFA: has been involved in data analysis and revising final manuscript, SL participated in designing of the study and in critical reading of manuscript, PI conceived of the study, has been involved in Cy5TLP's: viroplasms colocalization, interpretation of results and drafted the manuscript. All authors read and approved the final manuscript.

\section{Competing interests}

The authors declare that they have no competing interests.
Received: 9 September 2010 Accepted: 29 November 2010 Published: 29 November 2010

\section{References}

1. Estes MK, Kapikian AZ: Rotaviruses. In Fields Virology. Volume 2. Edited by: Knipe NM, Howley PM. Philadelphia: Lippincott Williams @Wilkins, a Wolters Kluwer Business; 2007:1917-1974.

2. Petrie BL, Greenberg HB, Graham DY, Estes MK: Ultrastructural localization of rotavirus antigens using colloidal gold. Virus Res 1984, 1:133-52.

3. Knipe DM, Samuel CE, Palese P: Virus-host cell interactions. In Fields Virology. Volume 1.. 4 edition. Edited by: Knipe DM, Howley PM. Philadelphia: Lippincott-Raven; 2001.

4. Becker MM, Goral MI, Hazelton PR, Baer GS, Rodgers SE, Brown EG, Coombs KM, Dermody TS: Reovirus sigmaNS protein is required for nucleation of viral assembly complexes and formation of viral inclusions. J Virol 2001, 75:1459-75.

5. Fabbretti E, Afrikanova I, Vascotto F, Burrone OR: Two non-structural rotavirus proteins, NSP2 and NSP5, form viroplasm-like structures in vivo. J Gen Virol 1999, 80:333-9.

6. Campagna M, Budini M, Arnoldi F, Desselberger U, Allende JE, Burrone OR: Impaired hyperphosphorylation of rotavirus NSP5 in cells depleted of casein kinase 1alpha is associated with the formation of viroplasms with altered morphology and a moderate decrease in virus replication. $J$ Gen Virol 2007, 88:2800-10.

7. Lopez T, Rojas M, Ayala-Breton C, Lopez S, Arias CF: Reduced expression of the rotavirus NSP5 gene has a pleiotropic effect on virus replication. $J$ Gen Virol 2005, 86:1609-17.

8. Silvestri LS, Taraporewala ZF, Patton JT: Rotavirus replication: plus-sense templates for double-stranded RNA synthesis are made in viroplasms. Journal of Virology 2004, 78:7763-74.

9. Arnoldi F, Campagna M, Eichwald C, Desselberger U, Burrone OR: Interaction of rotavirus polymerase VP1 with nonstructural protein NSP5 is stronger than that with NSP2. J Virol 2007, 81:2128-37.

10. Gonzalez RA, Espinosa R, Romero P, Lopez S, Arias CF: Relative localization of viroplasmic and endoplasmic reticulum-resident rotavirus proteins in infected cells. Arch Virol 2000, 145:1963-73.

11. Patton JT, Vasquez-Del Carpio R, Tortorici MA, Taraporewala ZF: Coupling of rotavirus genome replication and capsid assembly. Adv Virus Res 2007, 69:167-201.

12. Campagna M, Eichwald C, Vascotto F, Burrone OR: RNA interference of rotavirus segment 11 mRNA reveals the essential role of NSP5 in the virus replicative cycle. J Gen Virol 2005, 86:1481-7.

13. Altenburg BC, Graham DY, Estes MK: Ultrastructural study of rotavirus replication in cultured cells. J Gen Virol 1980, 46:75-85.

14. Eichwald C, Rodriguez JF, Burrone OR: Characterization of rotavirus NSP2/ NSP5 interactions and the dynamics of viroplasm formation. Journal of General Virology 2004, 85:625-34.

15. Cabral-Romero C, Padilla-Noriega L: Association of rotavirus viroplasms with microtubules through NSP2 and NSP5. Mem Inst Oswaldo Cruz 2006, 101:603-11.

16. Gonzalez RA, TorresVega MA, Lopez S, Arias CF: In vivo interactions among rotavirus nonstructural proteins. Archives Of Virology 1998, 143(5):981-996.

17. Dector MA, Romero P, Lopez $S$, Arias CF: Rotavirus gene silencing by small interfering RNAs. EMBO Rep 2002, 3:1175-80.

18. Zarate S, Espinosa R, Romero P, Mendez E, Arias CF, Lopez S: The VP5 domain of VP4 can mediate attachment of rotaviruses to cells. J Virol 2000, 74:593-9.

19. Guerrero CA, Zarate S, Corkidi G, Lopez S, Arias CF: Biochemical characterization of rotavirus receptors in MA104 cells. J Virol 2000, 74:9362-71.

20. Charpilienne A, Nejmeddine M, Berois M, Parez N, Neumann E, Hewat E, Trugnan G, Cohen J: Individual rotavirus-like particles containing 120 molecules of fluorescent protein are visible in living cells. J Biol Chem 2001, 276:29361-7.

21. van der Schaar HM, Rust MJ, Chen C, van der Ende-Metselaar H, Wilschut J, Zhuang $X$, Smit JM: Dissecting the cell entry pathway of dengue virus by single-particle tracking in living cells. PLOS Pathog 2008, 4:e1000244.

22. Brandenburg B, Lee LY, Lakadamyali M, Rust MJ, Zhuang X, Hogle JM: Imaging Poliovirus Entry in Live Cells. PLoS Biol 2007, 5:e183. 
23. Pelkmans $L$, Kartenbeck J, Helenius A: Caveolar endocytosis of simian virus 40 reveals a new two-step vesicular-transport pathway to the ER. Nat Cell Biol 2001, 3:473-83.

24. Rust MJ, Lakadamyali M, Zhang F, Zhuang X: Assembly of endocytic machinery around individual influenza viruses during viral entry. Nat Struct Mol Biol 2004, 11:567-73.

25. Mossel EC, Ramig RF: Rotavirus genome segment 7 (NSP3) is a determinant of extraintestinal spread in the neonatal mouse. J Virol 2002, 76:6502-9.

26. Offit $P A$, Blavat $G$, Greenberg HB, Clark HF: Molecular basis of rotavirus virulence: role of gene segment 4. J Virol 1986, 57:46-9.

27. Haddow J, Clark B, Ni Y, Desselberger U: Biological function of the rotavirus protein VP4: observations on porcine isolates from China. Med Microbiol Immunol 1989, 178:163-76.

28. Sen A, Feng N, Ettayebi K, Hardy ME, Greenberg HB: IRF3 inhibition by rotavirus NSP1 is host cell and virus strain dependent but independent of NSP1 proteasomal degradation. J Virol 2009, 83:10322-35.

29. Martin D, Duarte $M$, Lepault J, Poncet D: Sequestration of free tubulin molecules by the viral protein NSP2 induces microtubule depolymerization during rotavirus infection. J Virol 84:2522-32.

30. Parker JS, Broering TJ, Kim J, Higgins DE, Nibert ML: Reovirus core protein mu2 determines the filamentous morphology of viral inclusion bodies by interacting with and stabilizing microtubules. J Virol 2002, 76:4483-96.

doi:10.1186/1743-422X-7-350

Cite this article as: Carreño-Torres et al.: Characterization of viroplasm formation during the early stages of rotavirus infection. Virology Journal 2010 7:350.

\section{Submit your next manuscript to BioMed Central and take full advantage of:}

- Convenient online submission

- Thorough peer review

- No space constraints or color figure charges

- Immediate publication on acceptance

- Inclusion in PubMed, CAS, Scopus and Google Scholar

- Research which is freely available for redistribution

Submit your manuscript at www.biomedcentral.com/submit 